\title{
Study on Color Analyzer based on the Multiplexing of TCS3200 Color Sensor and Microcontroller
}

\author{
Li Qiaoyi, Xiong Yanling, Yang Wenlong, Han Junsheng and Liang Huan \\ College of Applied Science, Harbin University of Science and Technology, \\ Harbin, China \\ xyling1964@163.com
}

\begin{abstract}
In consideration of the increasingly higher demand for color detection in the modern industry and agriculture, this paper presents and implements one kind of color analyzer based on TCS3200 color sensor and microcontroller AT89C52, which can readdirectly the color information and achieve the intelligent control. The color analy er consists of the lighting module, the color recognition module, the data processing module and the display module. The intelligent color analyzers having the advantages of high performance, low cost, easy operation and maintenance small size and good development can meet the requirements of practical application

Keywords: color sensors, TCS3200, AT89C52

\section{Introduction}

Currently the accuracy of the color recognition and detection is becoming increasingly important in the industry $[1,2]$. The method of measuring the color mainly contains relatively primitive visual measurement and advanced measurement instruments. The measurement instruments according to the different methods that the three primary colors obtained are divided into tyo categories; optical integral spectral color measurement instruments and color measurement instrument. And many types of color analyzers based on the colorimetry theory is produced and commoditized, but the existing measuring instruments on the market are not universal and have high cost and single function only [3-7].

This paper proposes a color analyzer based on TCS3200 color sensor and microcontroller AT89652 The TCS3200 color sensor is employed to collect the sample color information which can convert three primary color signals of the sample color into electrical frequencies and the output is the digital signal [7-9]. So the color analyzer can omit the operational amplifier modules and A / D converter circuit of the conventional color detection-system making the whole system more simple and compact. In particular, we use the microcontroller AT89C52 as the core control unit [10-12]. The LCD1604 serves as the display component. Its advantages of low cost, easy operation, good development and easy maintenance will have attractive applications in practical color measurement.
\end{abstract}

\section{Color Measurement Principle}

The color analyzer is composed of the lighting module, the color recognition module, the data processing module and the display module. The lighting module uses the lightemitting diode (LED) with high brightness white light as the light source of this analyzer; the color recognition module employs TCS3200 as the color sensor, realizing the early identification of the color information of the measured objects. 
TCS3200 color sensor launched by TAOS is a programmable color light-to-frequency converter. The silicon photodiode and the current-frequency converter are integrated in a single CMOS circuits with red, green and blue (RGB) filters. Figure 1 is the pin package and the functional block diagram of TCS3200.

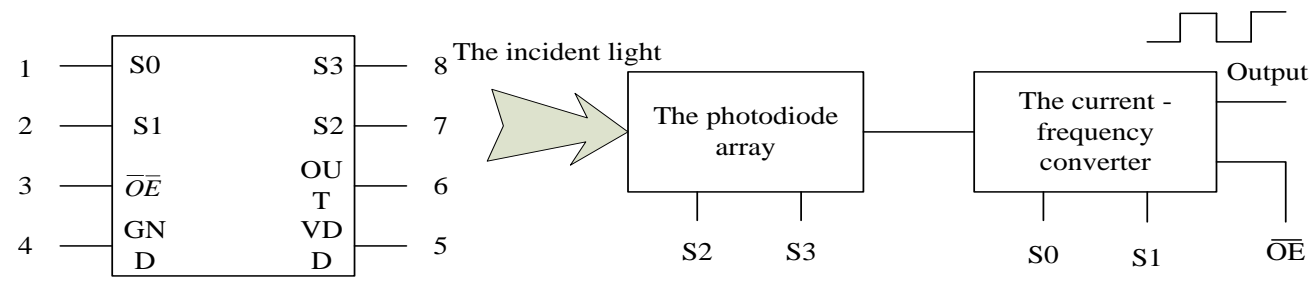

\section{Figure 1. The Pin Package and the Functional Block Diagram of TCS3200}

TCS3200 color sensor has four filter types. When the incident light irradiates the TCS3200 color sensor, different filters can be gated by controlling the different combinations of pin S2, S3, as shown in Table 1. The square wayes at different frequencies are output after the conversion of the current int the frequency, different colors and light intensities correspond to the square wave/at different frequencies. The typical output frequency range is $2 \mathrm{~Hz} \sim 500 \mathrm{kHz}$, using the programmable pin S0, S1 of TCS3200 to select the type of output scale factor, as shown in Table 2.

Table 1. TCS3200 Filter Type Selection Table 2.TCS3200 Output Scale

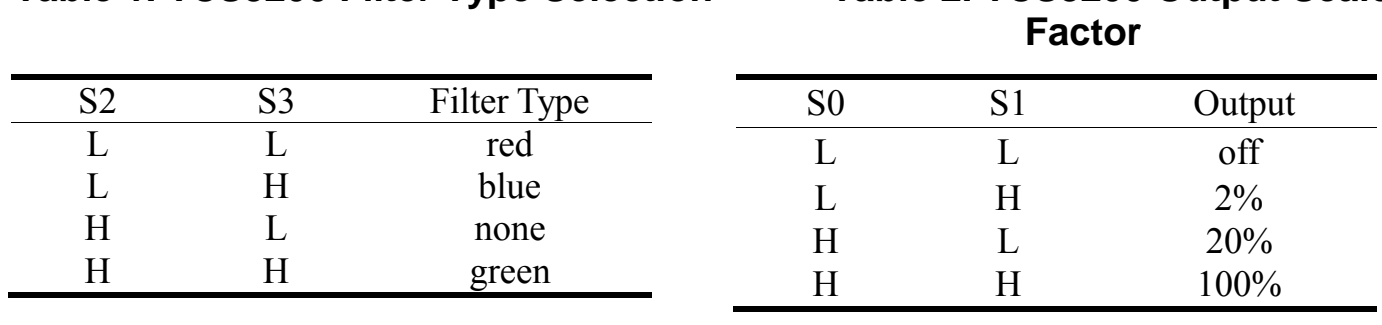

From three primary colors principle we know that the sample color can be obtained through the converter when the values of the primary colors are known [13-15]. The TaS3200 ean only make the one color pass when a color filter is selected, so the values ol three primary colors can be obtained in turn by gating different color filters the color value of the light irradiated to TCS3200 color sensor can be calculated by them.

The sensitivities of TCS3200 color sensor to three primary colors are different causing that the RGB output are not equal when TCS3200 color sensor measures the white light so the white balance needs to be conducted before the measurement [16]. The pecific method: the sensor is put on the bottom of an empty test tube, a white hight source is placed at the top of the test tube so that the light irradiated to TCS 3200 color sensor passes through the tube; then the green, blue and red filters are gated successively to measure the green, blue and red values, so the three adjustment parameters are obtained by the calculation. When TCS3200 color sensor is used to identify the color, we use three measured parameters to adjust the values of three primary colors. There are two methods to calculate the adjustment parameters. The first, the color filters are successively gated, the output pulses are counted until the time reaches 255 to stop for each filter, and the cost time of each color filter is calculated. These time must base on the time tested during the actual use of TCS3200 color sensor, the number of pulses during this measured time corresponds to the values of $\mathrm{R}, \mathrm{G}$ and $\mathrm{B}$. The second, the timer is set to a fixed time (e.g., 10ms), then three color filters are gated in turn, we calculate the pulse values 
of TCS3200 color sensor output during this time, so the scale factor can be calculated, we can become the measured pulses number to 255 by this scale factor. Therefore, the corresponding values of $\mathrm{R}, \mathrm{G}$ and $\mathrm{B}$ can be obtained by using the same time for counting the pulses number multiplied by the measured scale factor in the actual test.

\section{Hardware Design and Principle}

The overall design diagram of the color analyzer is shown in Figure 2. The data processing module based on the AT89C52 and the serial communication technology realizes the data communication with the host computer; the display module chooses the LCD to display the color information of the measured object [17].

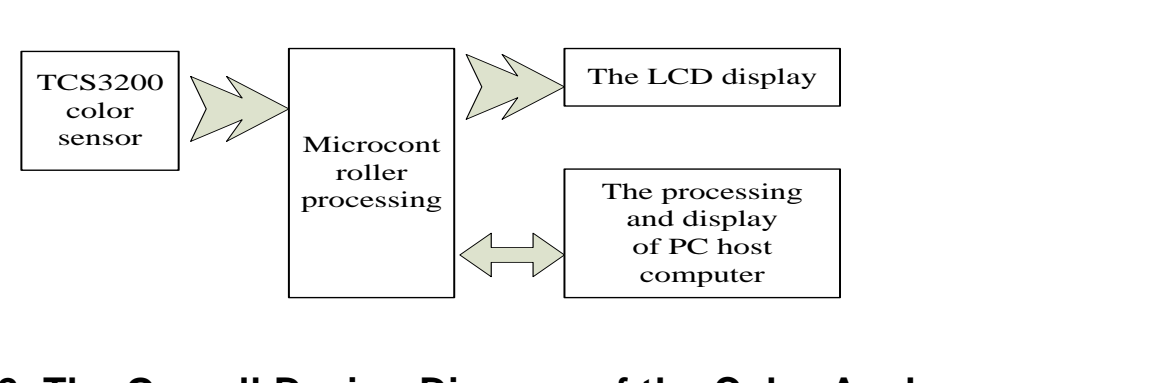

Figure 2. The Overall Design Diagram of the Color Analyzer

AT89C52 is a low-voltage, high-performance 8-bit intelligent control microcontroller. To ensure the stable operation of AT89C52, a reset circuit and a crystal circuit are built, as shown în Figure 3. Reset circuit enables the AT89C52 to return the initial state; the ctystal circuit forming a self-excited oscillator outputs the clock signal, when the pulse signal inpots the clock circuit of AT89C52, the clock of the master control of AT89C52 can be formed to enable AT89C52 to work normally.

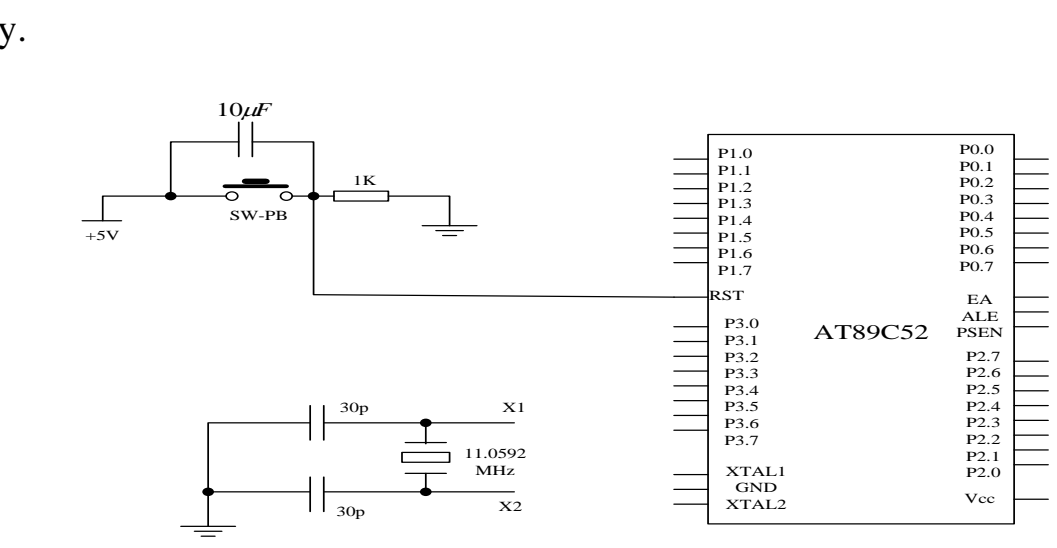

\section{Figure 3. The Wiring Diagram of the Reset Circuit and the Crystal Circuit}

In order to achieve the data collected by the color analyzer to be processed and displayed on the PC, the AT89C52 serial port and PC serial communication circuit are built. Taking into account the inconsistency of the output data information level processed by AT89C52 and the level of PC host computer, MAX232 is used to convert the levels between them. The pin and the function of MAX232 and the serial communication circuit based on it as the main component are shown in Figure 4. 


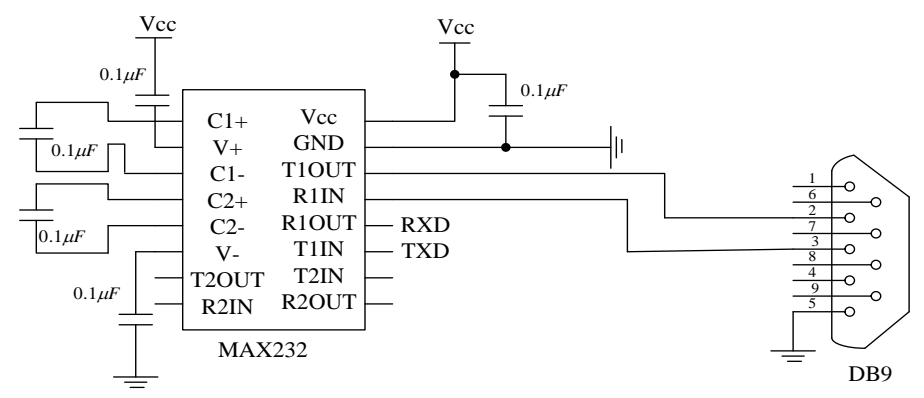

Figure 4. The Serial Communication Circuit based on MAX232

In the analyzer system, the keyboard functions include: the white balance adjustment, the output of standard color sampling, clear data send data, etc. The number of keys is a little big in the design, so a matrix keyboard is adopted to improve the work efficiency of AT89C52 and ensure that no matter which button is pressed, an external interrupt source input will generate only once. This will not affect the multiple interrupt sources simultaneously taken up and reduce the waste of CPU resources. The specific circuit is shown in Figures.

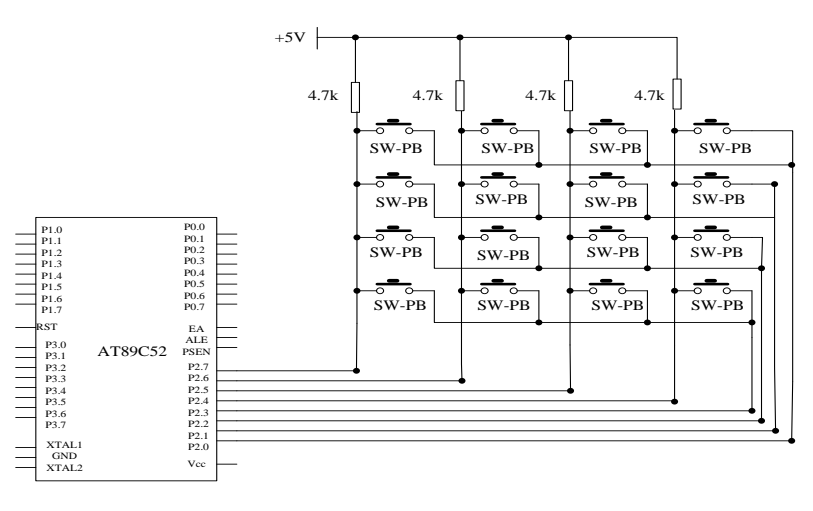

Figure 5. The wiring Diagram of the Matrix Keyboard Interface and AT89C52

LCD has the characteristics of small size, low power consumption and high immunity to electromagnetic interference so on. Therefore, we choose LCD1604 with 4-line display as the display module. The wiring diagram of LCD and AT89C52 is shown in Figure 6.

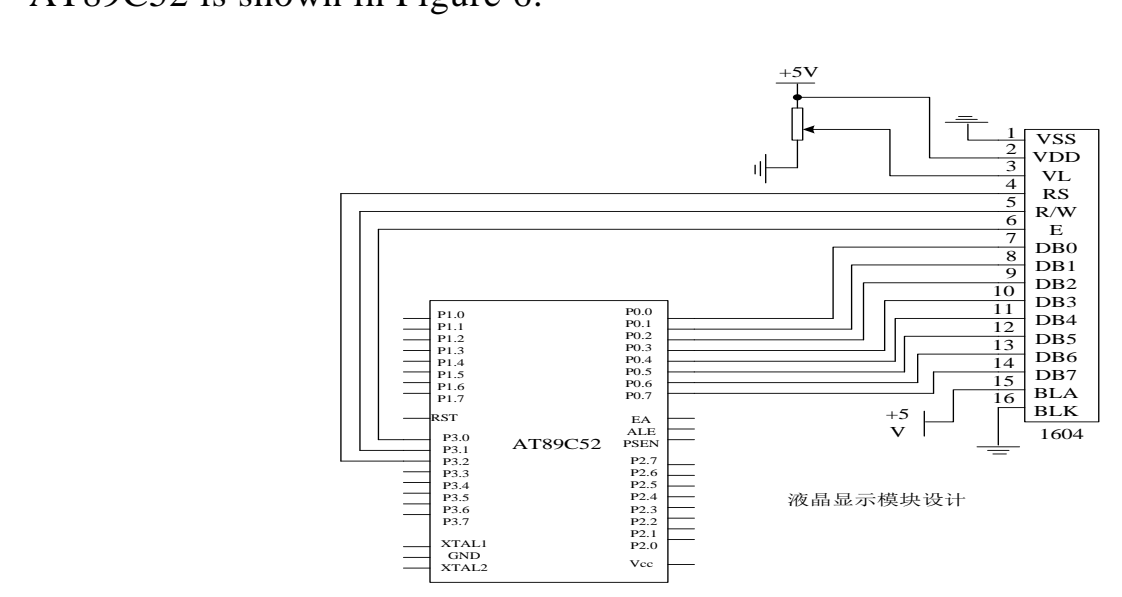

Figure 6. The Wiring Diagram of LCD and AT89C52

The wiring circuit of the overall hardware simulation is shown in Figure 7. 


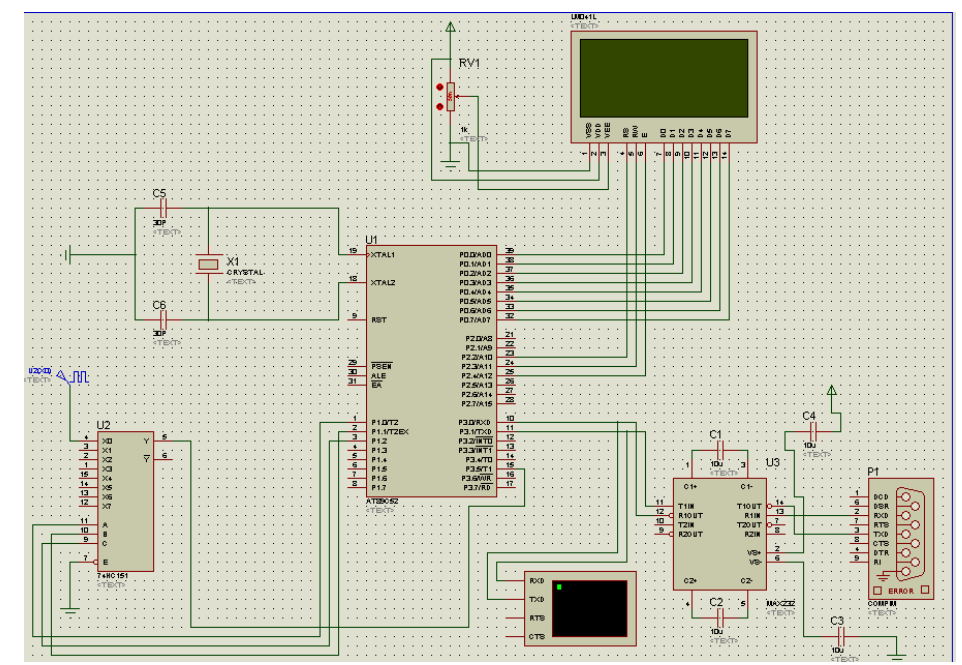

Figure 7. The Simulation Diagram of the Color Recognition Analyzer

\section{Software Design and Implementation}

PC software written primarily is used to read, display and save the color data information of the measured sample processed by the microcontroller; meanwhile PC software issues the command achieving the intelligent control on the microcontroller to control the working stat is of the microcontroller. In this paper, VB language is applied to compile the PC software. The PC software includes five modules of the serial control settings, switches, data acquisition, data processing and display. Specific PC interface is Shown in Figure 8.

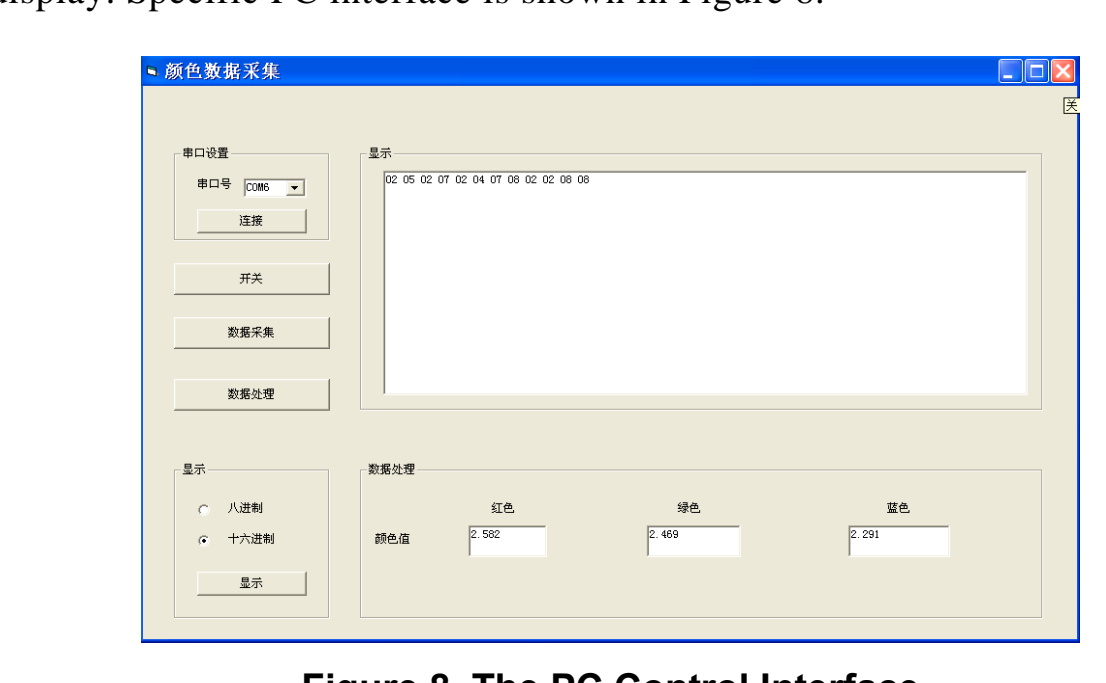

Figure 8. The PC Control Interface

The main role of AT89C52 is to complete the control on the color sensor picking up the sample color information, the operating state of MAX232 serial circuit and LCD display based on the command sent from the host computer, and process primarily and synchronously the three primary colors data. The collected data stored in the internal memory of the AT89C52r is sent to the host computer according to PC software commands. In this paper, the software program of AT89C52 is implemented by $\mathrm{C}$ language.

The software flowchart of the whole measurement system is shown in Figure 9. 


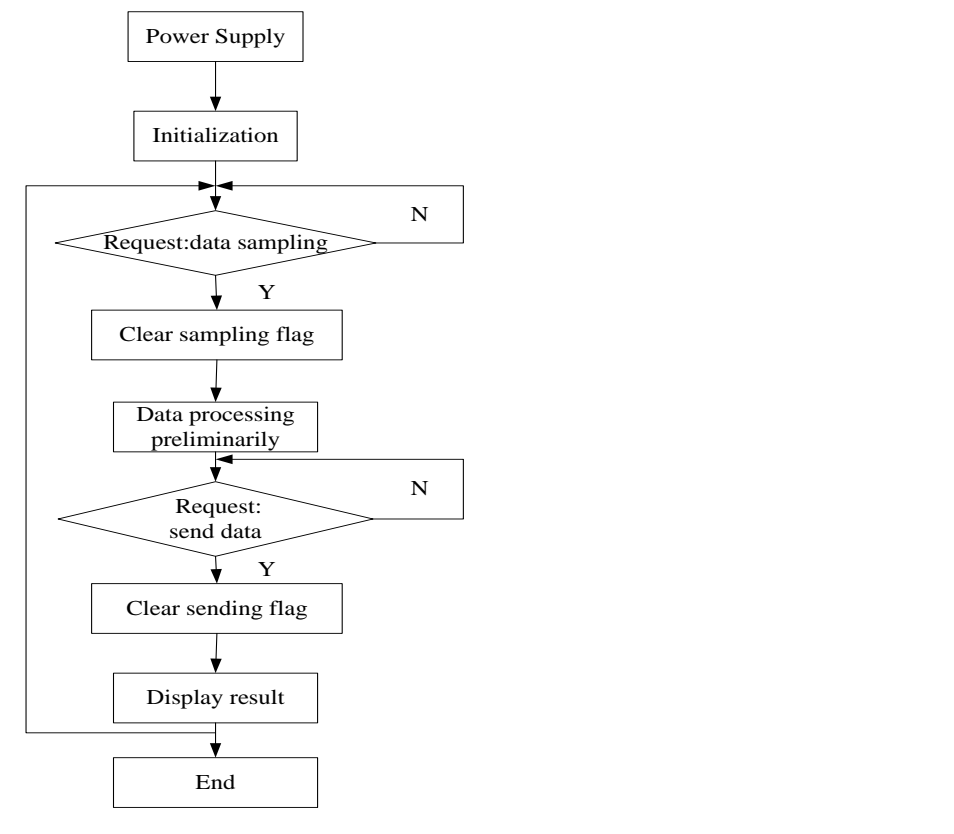

Figure 9. The Software Flowchart of the Whole Measurement System

Based on the overall works above, the progran is burned mto the core control chip -AT89C52 by STC software after the hardware is butlt. Then the color analyzer can achieve color detection recognition and detection after the power is supplied to the system is shown in Figure 9. The actual operation condition of the color analyzer is shown in Figure 10.

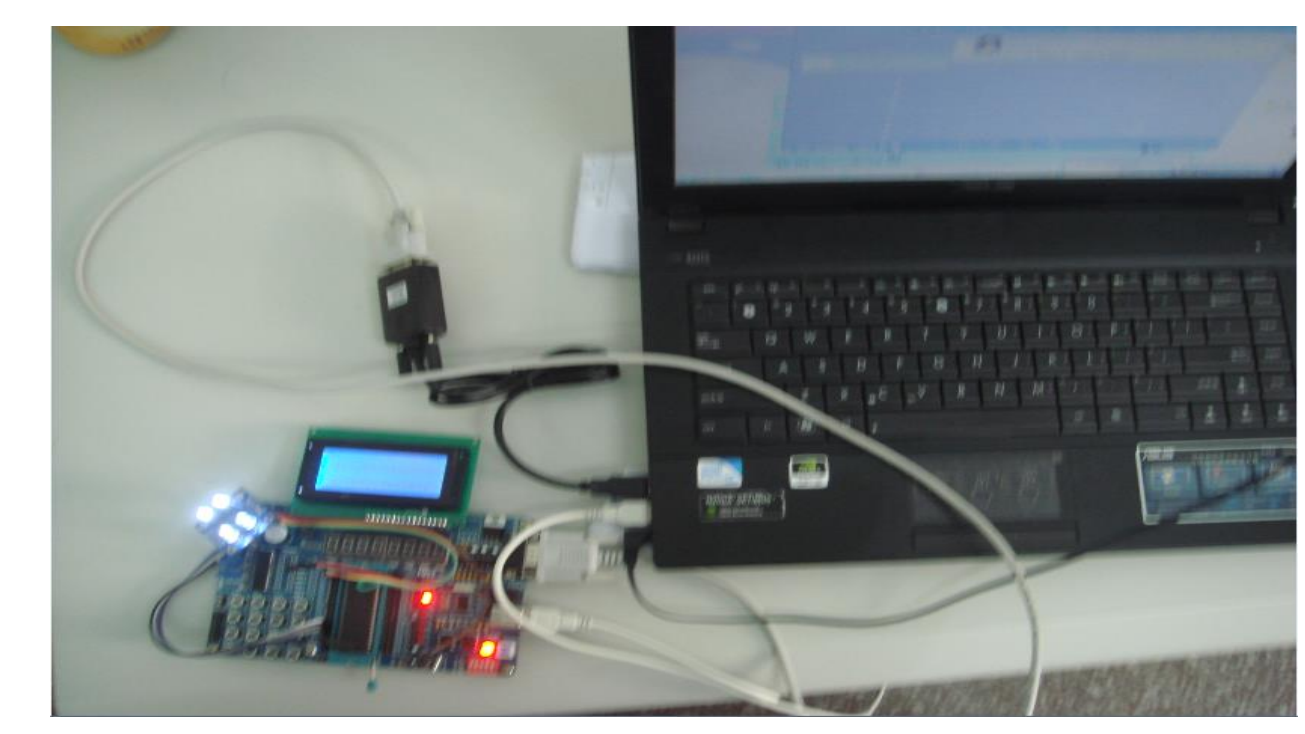

Figure 10. The Actual Operation Condition of the Color Analyzer 


\section{Conclusion}

This paper proposes and carries out a color analyzer based on TCS3200 color sensor and microcontroller. The hardware configuration module including power, lighting system, photoelectric detection system, data acquisition and processing module and display module and software design are achieved, respectively. The system has high performance, low cost, small size, etc. and the software is easy to develop and optimize.

\section{References}

[1] Rohner, Ernst and Stoger, "Color quality assurance with instrumental color measurement", Industrial \& production engineering(1989), vol. 13, pp. 80-81.

[2] K. Liang, W. Li and H. R. Ren, "Color measurement for RGB white LEDs in solid-state lighting using a BDJ photodetector", Displays, vol. 30, (2009), pp. 107-113.

[3] J. Chen, The development of the TCA - 20 type CRT color analyzer, Television Technology, vol. 10, (2001), pp. 95-98.

[4] P. Haihong and C. Lin, B. Huang, and J. Meng, "The design of high-precision color recognit on system based on TCS230 color sensor", Microcomputer Information, vol. 16, (2009), pp. 159-161.

[5] X. Peng and B. Peng, "The research of color recognition system based on TCS230 color sensor combined with BP neural network", Food and Machinery, vol. 03, (2010), pp. 108-123.

[6] J. Hu, "TCS230 color sensor and the color recognition circuit" The Application of Microcontroller and Embedded System, vol. 04, (2006), pp. 40-41.

[7] Y. Jiang and Y. Deng, Design of Multipoint Color Measuring Device Based on TCS3200, Instrumentation Technology, vol. 03, (2011), pp. 54-57.

[8] J. Aji, "Object sorting robotic arm based on colour sensing", International Journal of Advanced Research in Electrical, Electronics and Instrumentation Engineerng, vol. 3, (2014), pp. 7741-7743.

[9] J. Liu, L. Yao and L. Zhang, "A solution color detection method based on TCS3200 sensor", Journal of Nanjing University, vol. 46, (2010), pp. 100-103.

[10] L. Wang, L. Xiu and J. Yu, "The design of sevial communication protocol based on PC and single chip microcomputer AT89C52", Mechanieal \& Electrical Engineering Technology, vol. 2, (2006), p. 19-21.

[11] Y. Zhang and Z. Zheng, "The data acquisition system based on single chip microcomputer AT89C52", Chemical Antromation and Instrument, Vol. 3, (2010) pp. 110-112.

[12]H. Yan, G. Liang and C. Hou, "The realization of serial communication of AT89C52 with VB", Microcomputer Information vol. 12, (2003), pp. 65-66.

[13] X. Zhao, Z. Fang and G. Mu, "Research on the colorimetric properties of LED projection light source", ACTA PHYSICA SINICA, vol. 56, (2007), pp. 2357-2359.

[14] F. Gao, L. Li and K. An, "The design of color identification system based on RGB, Sensors and Microsystems". (2012), vol. 10, pp 84-87.

[15]Z. Gao, B. Gao and J. Song, "The design of oil color detection system based on RGB color sensor", Process Automation Instrumentation, (2013), vol. 08, pp. 982-985.

[16] J. Yao, "The calibration of the colour analyzer", Computer \& Peripherals, vol. 03, (1997), pp. 30-32.

[17] Z. Zhang, M. Wan and Z. Zhang, „The design of parallel interface driver of LCD display module”, Foreign Electronie Measurement Technology, (2009), vol. 10, pp. 64-68. 
International Journal of Hybrid Information Technology

Vol.7, No.5 (2014)

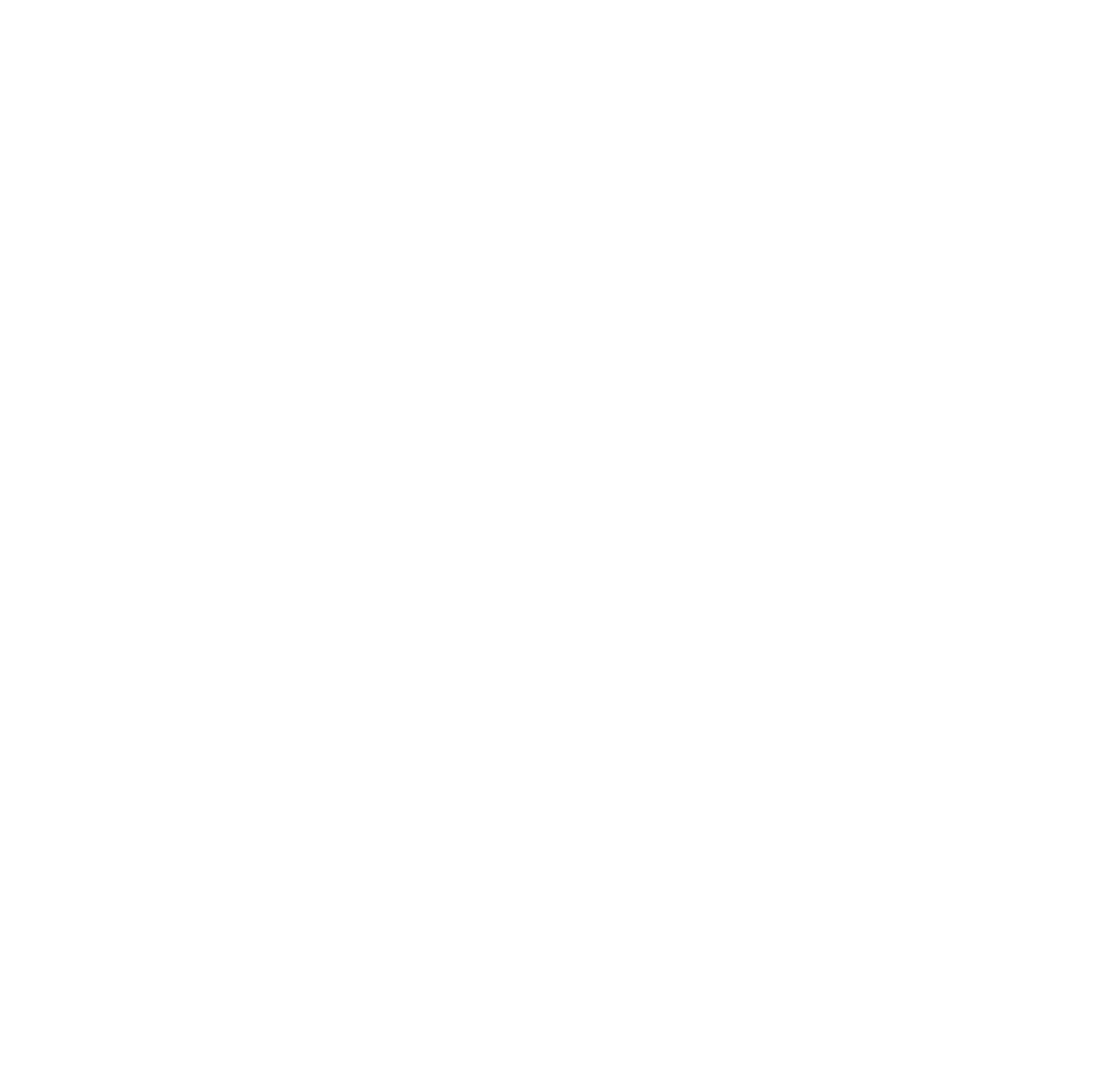

